Published in final edited form as:

Clin Lymphoma Myeloma Leuk. 2015 December ; 15(12): 766-770.e4. doi:10.1016/j.clml.2015.07.637.

\title{
Patterns of Venous Thromboembolism Prophylaxis During Treatment of Acute Leukemia: Results of a North American Web- Based Survey
}

\author{
Eun-Ju Lee ${ }^{1}$, B. Douglas Smith ${ }^{2}$, Jessica W. Merrey ${ }^{3}$, Alfred I. Lee ${ }^{1}$, Nikolai A. Podoltsev ${ }^{1}$, \\ Lisa Barbarotta ${ }^{1}$, Mark R. Litzow ${ }^{4}$, Thomas Prebet ${ }^{1}$, Selina M. Luger ${ }^{5}$, Steven Gore ${ }^{1}$, \\ Michael B. Streiff ${ }^{3}$, and Amer M. Zeidan ${ }^{1}$ \\ ${ }^{1}$ Section of Hematology, Department of Internal Medicine, Yale University, New Haven, CT \\ ${ }^{2}$ Department of Oncology, Johns Hopkins University, Baltimore, MD \\ ${ }^{3}$ Department of Medicine, Johns Hopkins University, Baltimore, MD \\ ${ }^{4}$ Department of Medicine, Mayo Clinic, Rochester, MN \\ ${ }^{5}$ Department of Medicine, University of Pennsylvania Medical Center, Philadelphia, PA
}

\begin{abstract}
Venous thromboembolism commonly occurs in patients with acute leukemia. We surveyed North American providers, and of the 151 responses, approximately half reported using pharmacologic anticoagulation during induction and consolidation treatment, while 15\% did not use prophylaxis and 36\% used mechanical methods and ambulation. These data highlight the need for further investigation and development of evidence-based guidelines for prophylaxis in this at-risk population.
\end{abstract}

Background-Venous thromboembolism (VTE) occurs in $2 \%$ to $12 \%$ of patients with acute leukemia (AL) despite disease- and therapy-associated thrombocytopenia, and it can be associated with significant morbidity and mortality. Because of the few high-quality studies, there are no evidence-based guidelines for VTE prophylaxis in this patient population. We sought to determine the spectrum of practice regarding prevention of VTE in patients with AL during induction and consolidation therapies.

Methods-We conducted a 19-question Web-based survey directed at North American providers caring for these patients. One hundred fifty-one of 215 responses received were eligible for analysis, with a response rate of $20.9 \%$ among physicians who treated leukemias.

Results-Overall, $47 \%$ and $45 \%$ of providers reported using pharmacologic VTE prophylaxis during induction and consolidation phases, respectively. Approximately $15 \%$ of providers did not

Address for correspondence: Amer M. Zeidan, MBBS, MHS, Section of Hematology, Department of Internal Medicine, Yale University, 333 Cedar Street, PO Box 208028, New Haven, CT 06520-8028, Fax: (203) 785-7232; amer.zeidan@ yale.edu.

Disclosure

The authors have stated that they have no conflicts of interest.

Supplemental Data

A supplemental figure accompanying this article can be found in the online version at http://dx.doi.org/10.1016/j.clml.2015.07.637. 
provide any VTE prophylaxis, while 36\% used mechanical methods and ambulation. Among providers who did not recommend pharmacologic prophylaxis, the most commonly cited reasons were the perceived high risk of bleeding (51\%), absence of data supporting use (38\%), and perceived low risk of VTE $(11 \%)$.

Conclusion-Large, prospective studies are needed to define the safest and most effective approach to VTE prevention in patients with AL.

\section{Keywords}

Acute leukemia; Acute lymphoid leukemia; Acute myeloid leukemia; Deep venous thrombosis; Patterns of practice

\section{Introduction}

Hematologic malignancies are associated with a significant risk of venous thromboembolism (VTE), comparable to that of higher-risk solid tumors including brain, pancreatic, and ovarian cancer. ${ }^{1}$ Studies of acute leukemia (AL) patients have demonstrated an incidence of VTE ranging from $2.2 \%$ to $12 \%$, with thrombotic events occurring before diagnosis, at the time of diagnosis, or during treatment. ${ }^{2-7}$ The pathogenesis of VTE in AL patients is multifactorial. Leukemic cells produce procoagulant, proteolytic, and fibrinolytic factors including tissue factor and cancer procoagulant. ${ }^{8-10}$ Blast cells secrete proinflammatory cytokines such as tumor necrosis factor alpha and interleukin $1 \beta$, whose downstream effects lead to a thrombotic tendency. ${ }^{8-10}$ Chemotherapy also contributes to a prothrombotic state via direct endothelial damage and destruction of leukemic cells as well as through liver injury causing reduced synthesis of natural anticoagulants (protein C, protein $\mathrm{S}$, antithrombin). ${ }^{8-10}$ In particular, L-asparaginase decreases production of antithrombin. ${ }^{10}$ Indwelling central venous catheters (CVC), immobility, infections, and use of high-dose steroids are other thrombotic risk factors. ${ }^{11}$

Given prolonged thrombocytopenia during induction and consolidation therapies, pharmacologic prophylaxis and treatment of VTE can be challenging. This underscores the importance of developing safe and effective methods of VTE prevention in this at-risk population. To date, there have been no large, prospective studies addressing VTE prophylaxis in AL patients, nor are there evidence-based guidelines to assist clinicians. On the basis of our experience and the lack of consensus guidelines, we hypothesized that there would be a wide range in provider practice regarding methods of VTE prevention in patients with AL. To determine the current individual practices in North America, we devised a Web-based survey of VTE prophylaxis practice among clinicians caring for patients with AL.

\section{Methods}

To determine current practices of VTE prophylaxis among health care providers, we devised an anonymous 19-question Web-based survey (Supplemental Figure 1 in the online version) utilizing SurveyMonkey software (http://SurveyMonkey.com/). The survey was approved by the Johns Hopkins institutional review board and distributed by e-mail to members of 
Eastern Cooperative Oncology Group-American College of Radiology Imaging Network (ECOG-ACRIN) centers on October 22, 2014, by the ECOG-ACRIN Clinical Education and Awareness Team. Four reminders were sent at 2-week intervals, with the survey closing on December 8,2014 . The collected data were de-identified and stored on a password-protected computer and analyzed using descriptive statistics.

\section{Results}

The survey was distributed to an e-mail list that had 6446 recipients, of whom 3628 were physicians. Of those physicians, 723 have treated leukemia patients. We received a total of 215 responses and included responses only from clinicians who reported directly managing care of leukemia patients. Of the total 215 respondents, 64 were excluded for the following reasons: 52 respondents did not directly manage medical care of AL patients, 3 referred AL patients to other centers, 5 left blank responses to all questions regarding VTE prophylaxis, 1 was a duplicate entry, and 3 described their position as support or office staff.

On the basis of the 151 analyzed responses, the response rate for all physicians was $4.2 \%$ and the response rate for physicians who treat leukemia was $20.9 \%$. The final sample included 151 responses from clinicians based in the United States $(\mathrm{n}=147)$ and Canada $(\mathrm{n}=$ 4), representing 88 different institutions and 37 states or provinces. Sixty-four percent of respondents were men, and $84 \%$ were between the ages of 31 to 60 years. Ninety-four percent were board certified in hematology and/or oncology. The complete characteristics of the survey population are listed in Table 1.

Provider patterns of VTE prophylaxis for AL during induction and consolidation therapies excluding acute promyelocytic leukemia and those with disseminated intravascular coagulation are illustrated in Figure 1. The survey questions addressing methods of VTE prophylaxis provided choices of no prophylaxis, ambulation, compression devices (graduated compression stockings or intermittent pneumatic compression devices), and pharmacologic anticoagulation, and allowed respondents to choose as many options as applicable. Although the overall distribution of prophylaxis practice remained consistent between induction and consolidation, 18 providers indicated use of pharmacologic prophylaxis during induction but not during consolidation therapy, whereas 14 providers indicated the reverse practice.

Among the 86 providers providing pharmacologic prophylaxis during induction and/or consolidation, $60 \%$ designated $50,000 / \mu \mathrm{L}$ as the platelet count threshold below which they would hold prophylaxis and $26 \%$ used a platelet count of $30,000 / \mu \mathrm{L}$. Fewer providers chose a platelet count of $20,000 / \mu \mathrm{L}(4 \%), 75,000 / \mu \mathrm{L}(4 \%)$, and $100,000 / \mu \mathrm{L}(2 \%)$, whereas $2 \%$ held prophylactic anticoagulation only in the setting of active bleeding and $2 \%$ reported variable thresholds. A total of 94 providers did not use pharmacologic prophylaxis or chose to provide anticoagulation in either induction or consolidation but not in both. To assess reasons for withholding prophylactic anticoagulation, the survey offered the following choices: "risk of VTE is low," "risk of bleeding is high," and "absence of data supporting efficacy of VTE prophylaxis in these populations," again with the ability to choose all options that applied. Among providers not consistently providing pharmacologic 
prophylaxis, the most commonly cited reasons were the perceived high risk of bleeding $(51 \%)$ and the absence of data supporting its use (38\%). Eleven percent cited a perceived low risk of VTE.

There were more providers (59\%) who did not use pharmacologic VTE prophylaxis in induction therapy for patients with acute promyelocytic leukemia. Among the rest of the respondents, $16 \%$ held pharmacologic prophylaxis only during disseminated intravascular coagulation or thrombocytopenia (level not defined), $9 \%$ held prophylaxis only in the setting of an active bleed, $6 \%$ used prophylaxis in all patients, 5\% reported "NA," and 5\% provided other descriptive answers. Acute promyelocytic leukemia was addressed separately in the survey, given the concern for early hemorrhagic events in this disease.

\section{Discussion}

The findings of this study illustrate the wide variability in clinician practices of VTE prophylaxis for patients with AL during induction and consolidation therapies.

Approximately half of the providers surveyed relied on nonpharmacologic methods or did not use any prophylaxis, while the other half used pharmacologic VTE prophylaxis. A lack of consensus also exists regarding VTE prevention practices during induction and consolidation treatment and at what platelet threshold VTE prophylaxis should be held.

Although a proportion of providers indicated that they did not prescribe VTE prophylaxis because of the low risk of VTE, several studies have documented that VTE is not an infrequent complication in AL patients. A large retrospective study of 7876 patients with AL showed a 2-year cumulative incidence for VTE of 5.2\% in acute myeloid leukemia (AML) and $4.5 \%$ in acute lymphoblastic leukemia (ALL), with the majority of VTE events occurring in the first 3 months after diagnosis. ${ }^{3}$ A recently published retrospective study of 1295 patients with AL found an overall prevalence of VTE of $10.7 \%$. The majority of the VTEs occurred in the first 3 months of diagnosis and were upper extremity catheter related deep vein thrombosis. ${ }^{6}$ In a smaller observational cohort study of 379 patients with AL, VTE was the presenting clinical manifestation in $3.4 \%$ of the population. The cumulative overall incidence of thrombosis was 6.3\%. ${ }^{4}$ Similarly, in another small prospective study of 114 patients with AL, VTE was the presenting symptom in $3.5 \%$ of cases. ${ }^{5}$

These studies illustrate the hypercoagulability of leukemia itself, followed by the ongoing thrombotic risk associated with subsequent chemotherapy and indwelling CVC. Vu et al ${ }^{6}$ showed that most VTE events developed during induction chemotherapy in patients with AML, whereas ALL patients tended to develop VTE during consolidation chemotherapy. This disparity was attributed to institutional use of L-asparaginase in its ALL maintenance regimen, which is known to precipitate a hypercoagulable state by reducing antithrombin levels. Perhaps this, in addition to outpatient consolidation therapy for AML at some institutions, may explain why some survey respondents reported using prophylactic pharmacologic anticoagulation only during induction but not in consolidation therapy and vice versa. 
The most common concerns about using anticoagulation in VTE prophylaxis in AL were the lack of data supporting its use and the high risk of bleeding. Currently, there are no published prospective studies addressing VTE prophylaxis in patients with AL. Some data, however, are available from studies of thromboprophylaxis for CVC-related thrombosis. In a randomized, placebo-controlled multicenter trial of low-dose warfarin (1 $\mathrm{mg}$ daily) in cancer patients with a CVC, $24 \%$ of the patient population had AL. The study medication was held if platelet count was $20,000 / \mu \mathrm{L}$ or less and if there were many interruptions in treatment. Although the study did not demonstrate a reduction in the incidence of CVC-related thrombosis with low-dose warfarin, there were also no significant adverse effects. ${ }^{12}$ Similarly, several other CVC thromboprophylaxis trials showed no significant increase in bleeding in those receiving anticoagulation. ${ }^{7,13,14}$ Although the lack of increased bleeding events seems promising, extrapolation of these data is limited by small numbers of included AL patients and wide variation in choice, dose, and holding parameters of the anticoagulants.

Further data regarding the safety of anticoagulation may be gleaned from a retrospective study of 53 patients e including those with hematologic malignancies e with a platelet count $<50,000 / \mathrm{cmm}$ who received therapeutic anticoagulation for VTE. ${ }^{15}$ Although 15 patients required dose reduction, the overall bleeding rate was $9.4 \%$. This rate is comparable to the $6 \%$ (major) and $14 \%$ (any) bleeding rate observed in those treated with therapeutic-dose low-molecular-weight heparin for VTE in the CLOT trial of patients with active cancer, VTE, and a higher platelet count of $>75,000 / \mathrm{cmm} .{ }^{16}$ In addition, in an attempt to guide clinical care for cancer patients with thrombocytopenia and VTE, an expert opinion proposed use of full-dose low-molecular-weight heparin for acute VTE if platelets > $50,000 / \mu \mathrm{L}$, dose reductions for platelets $<50,000 / \mu \mathrm{L}$, and holding anticoagulation for platelets $<30,000 / \mu \mathrm{L} .{ }^{17}$

Again, although these data may suggest an acceptable risk of bleeding with carefully monitored anticoagulation, further investigation is required. The need remains for dedicated primary thromboprophylaxis trials in AL patients using standard VTE pharmacologic prophylaxis as well as prospective trials to determine the optimal management of VTE therapy in thrombocytopenic patients with AL. Prevention of VTE in AL is crucial, as treatment of VTE is challenging, given the prolonged and severe thrombocytopenia associated with AL treatment regimens.

Inherent limitations of this survey-based study are inclusion bias and a limited response rate. Our data are further limited by the relatively small number of questions and the restricted number of response choices included in the survey. Because longer surveys are associated with lower completion rates, we made a conscious decision to limit the number of questions in order to increase our response rate. Therefore, we were not able to determine if there are differences in opinion regarding prophylaxis in ALL versus AML patients or differences in the reasons behind prophylaxis during induction but not consolidation and vice versa.

Among clinicians using prophylactic anticoagulation, we were not able to determine dosing practices. 


\section{Conclusion}

Despite these limitations, our findings highlight the diversity of VTE prophylaxis patterns in North America and emphasize the need to develop standardized, evidence-based guidelines in this at-risk population. Current data confirm that patients with AL have significant risk for VTE and suggest the safety of low-dose anticoagulation despite thrombocytopenia. Ideally, future studies should investigate the efficacy of prophylactic pharmacologic anticoagulation, initiation of prophylaxis (ie, at diagnosis or at the time of treatment), course of prophylaxis (induction and/or consolidation), and clarify holding parameters (ie, platelet thresholds, international normalized ratio cutoffs). Given the difficulty in treating VTE during treatment of AL, devising safe and effective ways to prevent VTE is critical and is an important area in need of further investigation.

\section{Supplementary Material}

Refer to Web version on PubMed Central for supplementary material.

\section{Acknowledgments}

This study was conducted by the ECOG-ACRIN Cancer Research Group (Robert L. Comis, MD, and Mitchell D. Schnall, MD, PhD, Group Co-Chairs) and supported in part by Public Health Service grants CA180820, CA180826, CA180802, and CA180790, and the National Cancer Institute, National Institutes of Health, and the Department of Health and Human Services. Its content is solely the responsibility of the authors and does not necessarily represent the official views of the National Cancer Institute. The authors would like to thank Marissa Six and Ruth Lambersky of the Clinical Education and Awareness Team of ECOG-ACRIN for their help in the distribution of the survey and collection of data.

\section{References}

1. Falanga A, Marchetti M, Russo L. Venous thromboembolism in the hematologic malignancies. Curr Opin Oncol. 2012; 24:702-10. [PubMed: 23014188]

2. Ziegler S, Sperr WR, Knobl P, et al. Symptomatic venous thromboembolism in acute leukemia. Incidence, risk factors, and impact on prognosis. Thromb Res. 2005; 115:59-64. [PubMed: 15567454]

3. Ku GH, White RH, Chew HK, et al. Venous thromboembolism in patients with acute leukemia: incidence, risk factors, and effect on survival. Blood. 2009; 113:3911-7. [PubMed: 19088376]

4. De Stefano V, Sora F, Rossi E, et al. The risk of thrombosis in patients with acute leukemia: occurrence of thrombosis at diagnosis and during treatment. J Thromb Haemost. 2005; 3:1985-92. [PubMed: 16102104]

5. Melillo L, Grandone E, Colaizzo D, et al. Symptomatic venous thromboembolism and thrombophilic status in adult acute leukemia: a single-center experience of 114 patients at diagnosis. Acta Haematol. 2007; 117:215-20. [PubMed: 17237616]

6 . Vu K, Luong NV, Hubbard J, et al. A retrospective study of venous thromboembolism in acute leukemia patients treated at the University of Texas MD Anderson Cancer Center. Cancer Med. 2015; 4:27-35. [PubMed: 25487644]

7. Cortelezzi A, Moia M, Falanga A, et al. Incidence of thrombotic complications in patients with haematological malignancies with central venous catheters: a prospective multicentre study. Br J Haematol. 2005; 129:811-7. [PubMed: 15953009]

8. Falanga A, Rickles FR. Management of thrombohemorrhagic syndromes (THS) in hematologic malignancies. Hematology Am Soc Hematol Educ Program. 2007:165-71. [PubMed: 18024625]

9. Rickles FR, Falanga A, Montesinos P, et al. Bleeding and thrombosis in acute leukemia: what does the future of therapy look like? Thromb Res. 2007; 120(suppl 2):S99-106. [PubMed: 18023721] 
10. Falanga A, Marchetti M. Venous thromboembolism in the hematologic malignancies. J Clin Oncol. 2009; 27:4848-57. [PubMed: 19752334]

11. Wun T, White RH. Venous thromboembolism in patients with acute leukemia, lymphoma, and multiple myeloma. Thromb Res. 2010; 125(suppl 2):S96-102. [PubMed: 20434017]

12. Couban S, Goodyear M, Burnell M, et al. Randomized placebo-controlled study of low-dose warfarin for the prevention of central venous catheter-associated thrombosis in patients with cancer. J Clin Oncol. 2005; 23:4063-9. [PubMed: 15767639]

13. Niers TM, Di Nisio M, Klerk CP, et al. Prevention of catheter-related venous thrombosis with nadroparin in patients receiving chemotherapy for hematologic malignancies: a randomized, placebo-controlled study. J Thromb Haemost. 2007; 5:1878-82. [PubMed: 17723127]

14. Del Principe MI, Buccisano F, Maurillo L, et al. Infections increase the risk of central venous cathetererelated thrombosis in adult acute myeloid leukemia. Thromb Res. 2013; 132:511-4. [PubMed: 24090605]

15. Pemmaraju N, Kroll MH, Afshar-Kharghan V, et al. Bleeding risk in thrombocytopenic cancer patients with venous thromboembolism (VTE) receiving anticoagulation. ASH Ann Meet Abstr. 2012:3408.

16. Lee AY, Levine MN, Baker RI, et al. Low-molecular-weight heparin versus a coumarin for the prevention of recurrent venous thromboembolism in patients with cancer. N Engl J Med. 2003; 349:146-53. [PubMed: 12853587]

17. Saccullo G, Marietta M, Carpenedo M, et al. Platelet cut-off for anticoagulant therapy in cancer patients with venous thromboembolism and thrombocytopenia: an expert opinion based on RAND/UCLA appropriateness method (RAM). ASH Annu Meet Abstr. 2013:581. 


\section{Clinical Practice Points}

- VTE occurs commonly in patients with AL.

- There are currently no evidence-based guidelines for VTE prevention in AL.

- This survey illustrates the wide variety in current practice patterns for VTE prophylaxis among North American providers.

- Large, prospective studies are needed to define safe and effective ways to prevent VTE in this at risk population. 


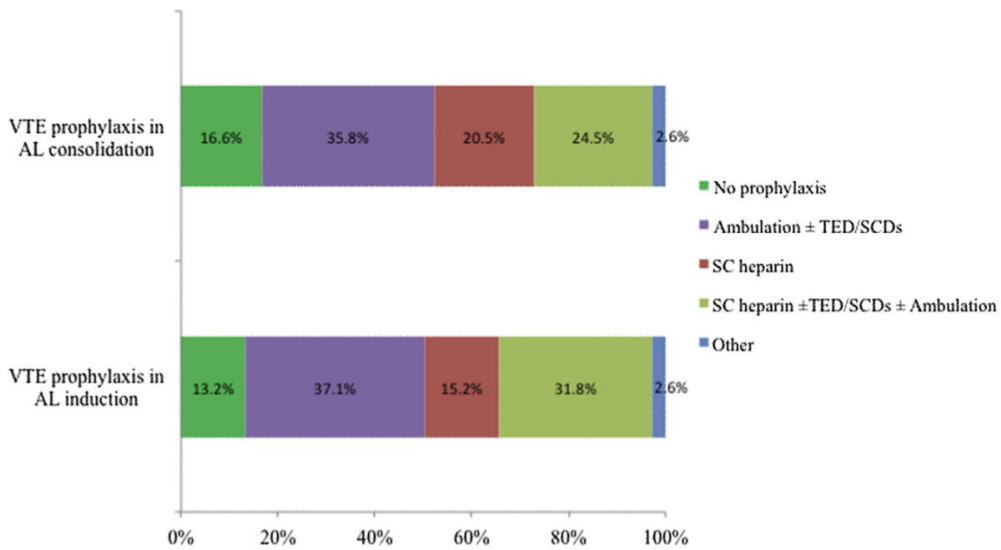

Figure 1.

Practice Patterns of VTE Prophylaxis for AL Patients During Induction and Consolidation Therapies

Abbreviations: $\mathrm{AL}=$ acute leukemia; $\mathrm{SC}=$ subcutaneous; $\mathrm{SCD}=$ sequential compression device; TED = thromboembolic deterrent stockings; VTE $=$ venous thromboembolism. 


\section{Table 1}

\section{Characteristics of Survey Respondents}

\begin{tabular}{|c|c|}
\hline Characteristic & n $(\%)$ \\
\hline \multicolumn{2}{|l|}{ Country of Practice } \\
\hline United States & $147(97)$ \\
\hline Canada & $4(3)$ \\
\hline \multicolumn{2}{|l|}{ Gender } \\
\hline Male & $96(64)$ \\
\hline Female & $55(36)$ \\
\hline \multicolumn{2}{|l|}{ Age } \\
\hline $20-30$ years & $1(1)$ \\
\hline $31-40$ years & $41(27)$ \\
\hline $41-50$ years & $46(30)$ \\
\hline $51-60$ years & $40(27)$ \\
\hline $61-70$ years & $18(12)$ \\
\hline $71-80$ years & $5(3)$ \\
\hline \multicolumn{2}{|l|}{ Job Description } \\
\hline Clinical researcher & $77(51)$ \\
\hline Clinician & $53(35)$ \\
\hline Clinical educator & $7(5)$ \\
\hline Clinician + researcher & $10(7)$ \\
\hline Translational researcher & $2(1)$ \\
\hline Basic science researcher & $2(1)$ \\
\hline \multicolumn{2}{|c|}{ Time After Fellowship Training } \\
\hline $0-5$ years & $38(25)$ \\
\hline $6-10$ years & $29(19)$ \\
\hline $11-20$ years & $34(23)$ \\
\hline 21 years or more & $45(30)$ \\
\hline Other & $5(3)$ \\
\hline
\end{tabular}

Clin Lymphoma Myeloma Leuk. Author manuscript; available in PMC 2015 December 01. 


\begin{tabular}{|c|c|}
\hline Characteristic & n $(\%)$ \\
\hline \multicolumn{2}{|l|}{ Type of institution of practice } \\
\hline University affiliated/public & $103(68)$ \\
\hline Private & $40(27)$ \\
\hline Veterans' administration & $2(1)$ \\
\hline Other & $6(4)$ \\
\hline \multicolumn{2}{|c|}{ No. of AL Patients Treated Yearly (Institution) } \\
\hline$<25$ & $35(23)$ \\
\hline $25-50$ & $29(19)$ \\
\hline $51-100$ & $33(22)$ \\
\hline $101-150$ & $34(23)$ \\
\hline$\geq 151$ & $16(11)$ \\
\hline Unsure & $4(3)$ \\
\hline \multicolumn{2}{|c|}{ No. of AL Patients Treated Yearly (Provider) } \\
\hline$<25$ & $74(49)$ \\
\hline $25-50$ & $55(36)$ \\
\hline $51-100$ & $15(10)$ \\
\hline $101-150$ & $4(3)$ \\
\hline$\unlhd 51$ & $3(2)$ \\
\hline \multicolumn{2}{|c|}{$\begin{array}{l}\text { Presence of VTE Prophylaxis Order Set for AL } \\
\text { Patients }\end{array}$} \\
\hline Yes & $36(24)$ \\
\hline No & $105(70)$ \\
\hline Unsure & $10(7)$ \\
\hline
\end{tabular}

Abbreviations: $\mathrm{AL}=$ acute leukemia; $\mathrm{VTE}=$ venous thromboembolism. 